Western University

Scholarship@Western

$7-5-2017$

Increased incidence of non-alcoholic fatty liver disease in male rat offspring exposed to fluoxetine during fetal and neonatal life involves the NLRP3 inflammasome and augmented de novo hepatic lipogenesis.

Nicole E De Long

Daniel B Hardy

daniel.hardy@schulich.uwo.ca

Noelle Ma

Alison C Holloway

Follow this and additional works at: https://ir.lib.uwo.ca/physpharmpub

Part of the Medical Physiology Commons, Pharmacy and Pharmaceutical Sciences Commons, and the Reproductive and Urinary Physiology Commons

Citation of this paper:

De Long, Nicole E; Hardy, Daniel B; Ma, Noelle; and Holloway, Alison C, "Increased incidence of nonalcoholic fatty liver disease in male rat offspring exposed to fluoxetine during fetal and neonatal life involves the NLRP3 inflammasome and augmented de novo hepatic lipogenesis." (2017). Physiology and Pharmacology Publications. 98.

https://ir.lib.uwo.ca/physpharmpub/98 


\title{
Increased incidence of non-alcoholic fatty liver disease in male rat offspring exposed to fluoxetine during fetal and neonatal life involves the NLRP3 inflammasome and augmented de novo hepatic lipogenesis
}

\author{
Nicole E. De Long ${ }^{1}$ | Daniel B. Hardy ${ }^{2}$ (1) | Noelle $\mathrm{Ma}^{2}$ | Alison C. Holloway ${ }^{1}$ (])
}

${ }^{1}$ Department of Obstetrics and Gynecology, McMaster University, Hamilton, Ontario, Canada, L8S 4K1

${ }^{2}$ Department of Obstetrics and Gynecology, Physiology and Pharmacology, University of Western Ontario, London, Ontario N6A 3K6

Correspondence

Alison Holloway, Department of Obstetrics \& Gynecology, McMaster University, RM HSC3N52, 1280 Main Street West, Hamilton,

Ontario, Canada, L8S 4K1.

Email: hollow@mcmaster.ca

Funding information

Canadian Institutes of Health Research, MOP111001 and MOP119323; CIHR Training Program in Reproduction, Early Development and the Impact on Health (REDIH)

\begin{abstract}
Up to $10 \%$ of women take selective serotonin reuptake inhibitors (SSRI) during pregnancy. Children exposed to SSRIs in utero have an increased risk of being overweight suggesting that fetal exposure to SSRIs can cause permanent metabolic changes. We have previously shown in rats that fetal and neonatal exposure to the SSRI antidepressant fluoxetine results in metabolic perturbations including increased hepatic triglyceride content; a hallmark of non-alcoholic fatty liver disease (NAFLD). Therefore, the aim of this study was to identify the mechanism(s) underlying the fluoxetine-induced increase in intrahepatic triglyceride content. Female nulliparous Wistar rats were given vehicle or fluoxetine $(10 \mathrm{mg} / \mathrm{kg} /$ day) orally for 2 weeks prior to mating until weaning. At 6 months of age, we assessed whether SSRI exposure altered components of the hepatic triglyceride biosynthesis pathway in the offspring and examined the molecular mechanisms underlying these changes. Male SSRI-exposed offspring had a significant increase in the steady-state mRNA levels of Elovl6 and Dgat1 and core components of the NLRP3 inflammasome (apoptosis-associated speck-like protein containing a caspase activation recruitment domain [ASC] and caspase-1). Augmented expression of Asc in the SSRI-exposed offspring coincided with increased histone acetylation in the proximal promoter region. Given that we have previously demonstrated that antenatal exposure to SSRIs can lead to fatty liver in the offspring, this raises concerns regarding the long-term metabolic sequelae of fetal SSRI exposure. Moreover, this study suggests that elevated hepatic triglyceride levels observed in the SSRI-exposed offspring may be due, in part, to activation of the NLRP3 inflammasome and augmentation of de novo lipogenesis.
\end{abstract}

\section{KEYWORDS}

developmental origins of disease, dyslipidemia, histone acetylation, inflammasome, metabolic syndrome, non-alcoholic fatty liver disease, post-translational histone modifications, selective serotonin reuptake inhibitors, triglycerides

\section{1 | INTRODUCTION}

Depression is currently estimated to affect as many as 840 million people worldwide (World Health Organization, 2008). Women of childbearing age appear to be particularly susceptible both to new onset depressive illness and the exacerbation of existing depression (Burt \& Stein, 2002; Noble, 2005). This vulnerability is particularly pronounced during the perinatal period. Indeed, the reported prevalence of perinatal depression ranges from 6.5\% to 25\% (Gavin et al., 2005; Gelaye,
Rondon, Araya, \& Williams, 2016). If left untreated, maternal depression has been linked to poor maternal and obstetrical outcomes (Gelaye et al., 2016; Grote et al., 2010; Marcus, 2009) and can have long-lasting deleterious effects on cognitive, behavioral and psychological development in the offspring (Stein et al., 2014). As a result, up to one in 10 women use antidepressants during pregnancy (Andrade et al., 2008; Cooper, Willy, Pont, \& Ray, 2007; Huybrechts et al., 2013). The most common antidepressants used during pregnancy are the selective serotonin reuptake inhibitors (SSRI); however, 
their long-term safety in the offspring remain elusive (Andrade et al. 2008; Muzik \& Hamilton, 2016; Smith et al., 2008; Trifirò et al., 2013; Wemakor, Casson, \& Dolk, 2014).

In children and adults the long-term use of SSRIs is associated with increased weight gain, dyslipidemia and new-onset type 2 diabetes (Jerrell, 2010; Yoon, Cho, Lee, \& Park, 2013; Schwartz et al., 2016;). As the fetus and neonate can be exposed to SSRIs via placental and lactational transfer (Rampono et al., 2009), it is plausible that SSRI use during pregnancy may also cause similar metabolic disturbances in the exposed offspring. In support of this hypothesis, Grzeskowiak et al. (2013) reported that male offspring exposed prenatally to an SSRI had an increased risk of being overweight by 7 years of age. Similarly, our lab has demonstrated in a rodent model that fetal and neonatal exposure to the SSRI antidepressant fluoxetine results in metabolic perturbations in adulthood including increased adiposity and insulin resistance (De Long et al., 2015). Moreover, fluoxetine exposure resulted in increased hepatic triglyceride content; a hallmark of nonalcoholic fatty liver disease (NAFLD) (De Long et al., 2015).

NAFLD is often associated with obesity and insulin resistance and is one of the most common chronic liver diseases worldwide (Bellentani, 2017). It encompasses a spectrum of stages which includes simple steatosis, non-alcoholic steatohepatitis (NASH; steatosis with evidence of hepatic inflammation with or without fibrosis) and cirrhosis (Tiniakos, Vos, \& Brunt, 2010). Accumulation of hepatic intracytoplasmic triglycerides is a key component of all stages of NAFLD (Tiniakos et al., 2010) and stems from an imbalance between lipid uptake, synthesis (i.e., de novo lipogenesis), utilization (i.e., fatty acid oxidation) and export (Tiniakos et al., 2010). Complex radiolabeling studies in humans have identified that an increase in de novo lipogenesis plays a significant role in intrahepatic triglyceride accumulation and the pathogenesis of NAFLD (Diraison, Moulin, \& Beylot, 2003; Donnelly et al., 2005; Softic, Cohen, \& Kahn, 2016). In vitro studies have demonstrated that fluoxetine augments lipid accumulation in hepatocytes in association with upregulation of genes critical for de novo lipogenesis (Feng et al., 2012; Xiong et al., 2014). This would suggest that the observed increase in hepatic triglycerides in fluoxetine-exposed offspring could be due to dysregulation of the same pathway (De Long et al., 2015). In addition to increased hepatic triglyceride accumulation, enhanced de novo lipogenesis also modifies fatty acid composition in the liver which in turn can lead to the development of NASH (Duwaerts \& Maher, 2014). The mechanism(s) underlying the progression of NAFLD to NASH are not well understood, but recent evidence from human studies and rodent models suggests that the NOD-like receptor family, pryin domain containing 3 (NLRP3) inflammasome may play a key role (Wan, Xu, Yu, \& Li, 2016).

The NLRP3 inflammasome is a large intracellular multiprotein complex consisting of nod-like receptor protein 3 (NLRP3), apoptosis-associated speck-like protein containing a caspase activation recruitment domain (ASC) and pro-caspase 1 (Schroder \& Tschopp, 2010). Activation of NLRP3 induces caspase-1 expression and the subsequent maturation of pro-interleukin-1 $\beta$ (pro-IL1 $\beta$ ) and pro-interleukin-18 (pro-IL18) into their active forms (Jo, Kim, Shin, \& Sasakawa, 2016; Schroder \& Tschopp, 2010). There is now convincing evidence that hepatic lipid accumulation is associated with increased expression and activation of the NLRP3 inflammasome (De Nardo \& Latz, 2011;
Haneklaus \& O'Neill, 2015). Moreover, in animal models, activation of the NLRP3 inflammasome has been shown to play a role in the progression of NAFLD to NASH (reviewed in [Wan et al., 2016]). This is of great interest as we have reported that fetal and neonatal exposure to fluoxetine increased the proportion of offspring with mild-to-moderate NASH (De Long et al., 2015). Moreover, as fluoxetine has been shown to increase production of IL1ß (Alboni et al., 2016), which is mainly dependent on activation of the NLRP3 inflammasome (Martinon, Burns, \& Tschopp, 2002), these data suggest that the increased prevalence of NASH in offspring born to fluoxetine-exposed dams may be a result of dysregulated de novo lipogenesis and activation of the NLRP3 inflammasome. Hence, the present study was designed to investigate the effects of fetal and neonatal exposure to fluoxetine on the transcriptional and epigenetic regulation of genes involved in hepatic de novo lipid synthesis and the NLRP3 inflammasome.

\section{2 | MATERIALS AND METHODS}

\section{1 | Maintenance and treatment of animals}

All animal experiments were approved by the Animal Research Ethics Board at McMaster University, in accordance with the guidelines of the Canadian Council for Animal Care. Nulliparous 200-250 g female Wistar rats (Harlan, Indianapolis, Indiana) were conventionally housed in polycarbonate microisolator cages under controlled lighting (12:12 L:D), humidity (40-50\%), and temperature $\left(22^{\circ} \mathrm{C}\right)$ with ad libitum access to water and standard chow diet (Teklad 22/5 rodent diet; Envigo, Indianapolis, Indiana). Dams were randomly assigned to receive vehicle ( $\mathrm{CON} ; \mathrm{N}=15$ ) or $10 \mathrm{mg} / \mathrm{kg} /$ day fluoxetine hydrochloride (FLX; $N=15$; Toronto Research Chemicals, Toronto, Ontario) orally in flavored gelatin cubes from 14 days prior to mating until weaning (postnatal day 21; PND21) as previously described (De Long et al., 2015). The dose of fluoxetine was chosen based on previous studies (Capello et al., 2011; Hui, Huang, Ebbert, \& Bina, 2007) to achieve serum fluoxetine levels in the rat which are representative of the median serum concentration of fluoxetine (i.e., $450 \mathrm{nmol} / \mathrm{L}$ ) reported in humans (Olivier et al., 2011; Sit, Perel, Helsel, \& Wisner, 2008). Dams were allowed to deliver normally. At birth (PND1), pups were weighed and sexed and litters were culled to 8 , preferentially selecting 4 male and 4 female offspring, to ensure uniformity of litter size between treated and control litters. Offspring were maintained on a chow diet until 26 weeks of age. We have previously reported that these offspring have increased hepatic triglyceride content, hepatic inflammation and an increase prevalence of NASH (De Long et al., 2015). At 26 weeks of age, one male offspring per litter was fasted overnight, euthanized by $\mathrm{CO}_{2}$ asphyxiation and liver tissue was collected and snap frozen in liquid nitrogen for subsequent analyzes. Only male offspring were analyzed in this study to prevent the confounding effects caused by the female reproductive cycle.

\section{2 | Transcriptional regulation of de novo lipogenesis and NLRP3 inflammasome}

To examine the effects of fetal and neonatal exposure to fluoxetine on de novo lipogenesis we performed qPCR to determine the 
steady-state mRNA expression of key enzymes responsible for the synthesis of triglycerides (acetyl-CoA carboxylase [Acc1], fatty acid synthase [Fasn], stearoyl CoA desaturase 1 [Scd1], ELOVL family member 6, elongation of very long fatty acid [Elovl6], and diacylglycerol acyltransferase 1 and 2 [Dgat1, Dgat2]). We also determined the steady-state mRNA levels of the core components of the NLRP3 inflammasome; nod-like receptor protein 3 (NIrp3), apoptosisassociated speck-like protein containing a caspase activation recruitment domain (Asc) and caspase-1 (Casp1).

Total RNA was isolated from male 26-week liver tissue using TRIzol ${ }^{\circledR}$ (Life Technologies, Grand Island, New York) extraction combined with the RNeasy ${ }^{\circledR}$ mini kit (Qiagen, Hilden, Germany) ( $N=9-10$ animals per treatment group). Complementary DNA (cDNA) was synthesized from $2 \mu \mathrm{g}$ of RNA using the High Capacity cDNA Reverse Transcription Kit (Applied Biosystems, Foster City, California) according to the manufacturer's instructions using PerfeCta ${ }^{\circledR}$ SYBR ${ }^{\circledR}$ green FastMix ${ }^{\circledR}$ (Quanta Biosciences, Gaithersburg, Maryland) on the LightCycler480 (Roche, Mississauga, Ontario). The cycling conditions included polymerase activation $\left(95^{\circ} \mathrm{C}\right.$ for $10 \mathrm{~min})$, followed by 40 cycles of denaturing $\left(95^{\circ} \mathrm{C}\right.$ for $\left.15 \mathrm{~s}\right)$ and annealing/elongation $\left(60^{\circ} \mathrm{C}\right.$ for $\left.1 \mathrm{~min}\right)$. The cycle threshold was set so that exponential increases in amplification were approximately level between all samples. Given all primer sets had equal priming efficiency, the $\Delta C$ t values for each primer set were obtained according to Livak and Schmittgen (2001), by normalizing gene expression to calibrated to two housekeeping genes (hypoxanthine-guanine phosphoribosyltransferase [Hprt] and Cyclophilin A [Cyc]). The average of all control Ct values, and the relative abundance of each primer set compared with calibrator was determined by the formula $2^{\Delta \Delta C t}$, in which $\Delta \Delta C t$ was the normalized value. Primer sequences are supplied in Table 1.

\section{3 | Epigenetic regulation of de novo lipogenesis and NLRP3 inflammasome}

\subsection{1 | Histone extraction}

Liver tissue collected from male offspring at 26 weeks of age was ground using a mortar and pestle and resuspended in in lysis buffer (10 mM HEPES, $\mathrm{pH} 7.9,1.5 \mathrm{mM} \mathrm{MgCl}, 10 \mathrm{mM} \mathrm{KCl}$, and protease cocktail inhibitor [Roche]). Hydrochloric acid was added to a final concentration of $0.2 \mathrm{M}$. The resultant solution was then sonicated at $30 \%$ amplitude for $5 \mathrm{~s}$ total, $1 \mathrm{~s}$ per pulse, and incubated on ice for $30 \mathrm{~min}$ with periodic vortexing. It was then centrifuged at $11000 \mathrm{~g}$ for $10 \mathrm{~min}$ at $4^{\circ} \mathrm{C}$. The supernatant was collected and underwent dialysis using Pierce Slide-A-Lyzer MINI Dialysis Units (3500 MWCO) (ThermoFisher Scientific, Burlington, Ontario, Canada). The order of dialysis occurred in the following manner: $2 \times 200 \mathrm{~mL}$ of $0.1 \mathrm{M}$ acetic acid for $1 \mathrm{~h}, 200 \mathrm{~mL}$ of deionized water for $1 \mathrm{~h}, 200 \mathrm{~mL}$ of deionized water for $3 \mathrm{~h}$, and $200 \mathrm{~mL}$ of water overnight. Samples were then stored at $-80^{\circ} \mathrm{C}$ until further analysis.

\subsection{2 | Global histone acetylation}

In total, $10 \mu \mathrm{g}$ of total histone proteins were loaded and subjected to $12 \%$ SDS-PAGE and transferred to a polyvinylidene difluoride (PVDF) blotting membrane (BioRad Laboratories, Hercules, California). Membranes were blocked for $3 \mathrm{~h}$ in 5\% (wt/vol) skim milk in TTBS (Tris-buffered saline [TBS], 0.1\% [vol/vol] Tween 20) at room temperature on a rocking platform and then incubated with Histone H3 (cat \#05-499; MilliporeSigma, Etobicoke, Ontario, Canada) and acetylated histone $\mathrm{H} 3[\mathrm{~K} 9,14]$ (cat \#sc-06-599; MilliporeSigma) primary antibody at $4^{\circ} \mathrm{C}$ overnight. After washing with TTBS, blots were incubated with peroxidase-conjugated secondary anti-rabbit or anti-mouse antibody (1:5000; GE Healthcare, QC, California) for $1 \mathrm{~h}$ at room temperature on a rocking platform. Blots were developed using enhanced chemiluminescence (ECL) (MilliporeSigma) and quantification was carried out using ImageLab 4.1 software (ImageLab Version 4.1 build 16; BioRad, Hercules, California). Immunoblots were subsequently incubated with stripping buffer (ThermoFisher Scientific) and re-probed with $\beta$ actin (1:4,000; Abcam, Toronto, Ontario, Canada) to control for protein loading. Histone $\mathrm{H} 3$ and acetylated histone $\mathrm{H} 3[\mathrm{~K} 9,14]$ was normalized to $\beta$-actin. Data presented are the ratio of acetylated histone $\mathrm{H} 3$ [K9,14]:Total histone H3.

\subsection{3 | Chromatin immunoprecipitation (ChIP)}

Chromatin was extracted from liver tissues excised at 26 weeks of age from male offspring as previously described (Sohi, Marchand, Revesz, Arany, \& Hardy, 2011). In brief, a small piece of snap frozen liver was homogenized and incubated in $0.5 \mathrm{~mL}$ of $1 \%$ formaldehyde for $10 \mathrm{~min}$ at room temperature to cross-link proteins and DNA. Glycine (0.125 M, final concentration) was added to all samples to terminate cross-linking. Samples were micro centrifuged at $950 \mathrm{~g}$ at room temperature for $5 \mathrm{~min}$ and supernatant was subsequently removed. The liver tissue was then washed once with cold phosphate-buffered saline before being placed in $500 \mu \mathrm{L}$ of DS lysis buffer (MilliporeSigma) with

TABLE 1 Real-time PCR rat primers used in the study

\begin{tabular}{|c|c|c|c|}
\hline Gene & Forward primer & Reverse primer & Genebank accession \# \\
\hline Acc1 & ATTGGGCACCCCAGAGCTA & CCCGCTCCTTCAACTTGCT & NM_022193.1 \\
\hline Asc & TGGTTTGCTGGATGCTCTGT & CACGAACTGCCTGGTACTGT & NM_172322.1 \\
\hline Casp1 & AACACCCACTCGTACACGT & TGAGGTCAACATCAGCTCCG & NM_012762.2 \\
\hline Dgat1 & CAGACCAGCGTGGGCG & GAACAAAGAGTCTTGCAGACGATG & NM_053437.1 \\
\hline Dgat2 & GGAACCGCAAAGGCTTTGTA & AATAGGTGGGAACCAGATCAGC & NM_001012345.1 \\
\hline Elovl6 & GGTCGGCATCTGATGAACAAG & CGAATATACTGAAGACCGCAAGAG & NM_134383.2 \\
\hline Nlrp3 & CACAACTCACCCAAGGAGGA & ACAGGCAACATGAGGGTCTG & NM_001191642.1 \\
\hline Scd1 & СTGACCTGAAAGCTGAGAAG & ACAGGCTGTGCAGGAAAGTT & NM_139192.2 \\
\hline
\end{tabular}


protease inhibitor cocktail (Roche). Each sample was sonicated to produce sheared, soluble chromatin. The lysates were diluted 10 times with the addition of ChIP dilution buffer (MilliporeSigma) and aliquoted to $300 \mu \mathrm{L}$ amounts. Each of the aliquots was precleared with protein A/G Plus agarose beads ( $40 \mu \mathrm{L}$; MilliporeSigma) and rotated for $2 \mathrm{~h}$ at $4^{\circ} \mathrm{C}$. To pellet the beads, samples were micro centrifuged at $20000 \mathrm{~g}$, and the supernatant containing the sheared chromatin was placed in new tubes. The aliquots were incubated with $3 \mu \mathrm{g}$ of acetylated histone $\mathrm{H} 3$ antibody (lysine 9,14, cat \#05-399; MilliporeSigma) and rotated overnight at $4^{\circ} \mathrm{C}$. Two aliquots were reserved as 'controls' - one incubated without anti- body ('input') and another with non-immune IgG (MilliporeSigma). Protein A/G Plus agarose beads $(60 \mu \mathrm{L})$ were added to each tube, the mixtures rocked for $1 \mathrm{~h}$ at $4^{\circ} \mathrm{C}$ and the immune complexes collected by centrifugation. The beads containing the immunoprecipitated complexes were washed sequentially for $5 \mathrm{~min}$ in wash buffer I $(20 \mathrm{mM}$ Tris- $\mathrm{HCl}$, pH 8.1,
$2 \mathrm{mM}$ EDTA, 0.1\% SDS, 1\% Tri- ton X-100, $150 \mathrm{mM} \mathrm{NaCl}$ ), wash buffer II (same as I, except containing $500 \mathrm{mM} \mathrm{NaCl}$ ), wash buffer III (10 mM Tris- $\mathrm{HCl}, \mathrm{pH}$ 8.1, 1 mM EDTA, 1\% NP-40, 1\% deoxycholate, $0.25 \mathrm{M} \mathrm{LiCl}$ ) and in $2 \times \mathrm{TE}$ buffer. The beads were eluted with $250 \mu \mathrm{L}$ elution buffer (1\% SDS, $0.1 \mathrm{mM} \mathrm{NaHCO} 3+20 \mu \mathrm{g}$ salmon sperm DNA [Sigma-Aldrich, Oakville, Ontario, Canada]) at room temperature. The elution step was repeated once and eluates were combined. Crosslinking of the immunoprecipitated chromatin complexes and 'input controls' ( $10 \%$ of the total soluble chromatin) was reversed by heating the samples at $65^{\circ} \mathrm{C}$ for $4 \mathrm{~h}$. Pro- teinase $\mathrm{K}(15 \mu \mathrm{g}$ [Invitrogen, Carlsbad, California]) was added to each sample in buffer (50 mM Tris- $\mathrm{HCl}, \mathrm{pH}$ 8.5, 1\% SDS, 10 mM EDTA) and incubated for $1 \mathrm{~h}$ at $45^{\circ} \mathrm{C}$. The DNA was purified by phenol-chloroform extraction and precipitated in ethanol overnight at $-20^{\circ} \mathrm{C}$. The supernatant was removed and pellets were dried. Both samples and 'input' controls' were diluted in 10-100 $\mu \mathrm{L}$ TE buffer prior to PCR analysis.

TABLE 2 Real-time PCR rat ChIP primers used in the study

\begin{tabular}{lll} 
Gene & \multicolumn{1}{c}{ Forward primer } & Reverse primer \\
\hline Asc & TGGAAGAGGGTGGAGTCCTG & CCGCGGTCACCTTTTACTCT \\
Casp1 & ACTCGGTACATGTGCCTCAC & AAAGGAGCAGGGAAACGATGT \\
Elovl6 & CCCACATGAGGGAAAGAGAG & CCAGCCCCACAGGTTTTT \\
Nlrp3 & AACTGTGCCAGTCTATGGGG & CTTAGATCCACGCTGGCAGA
\end{tabular}

(A)

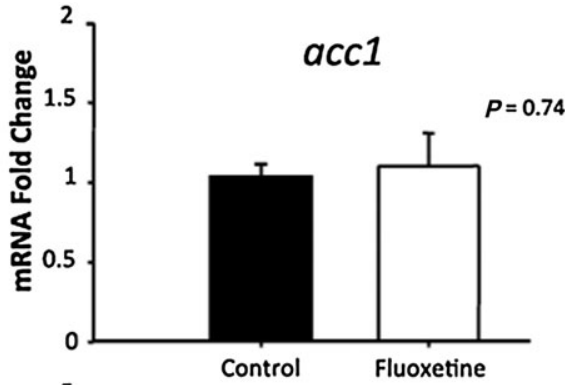

(C)

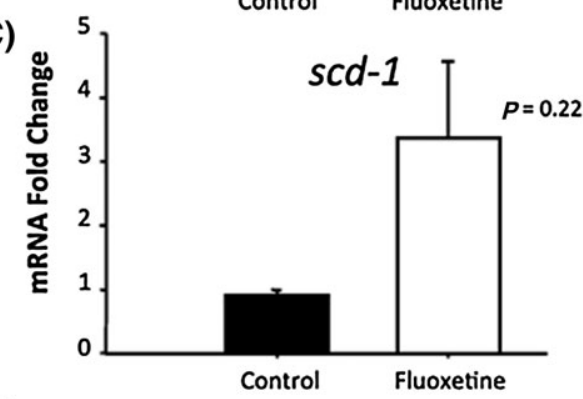

(E)

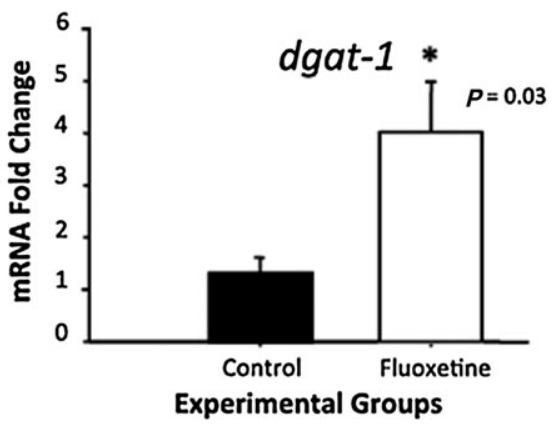

(B)

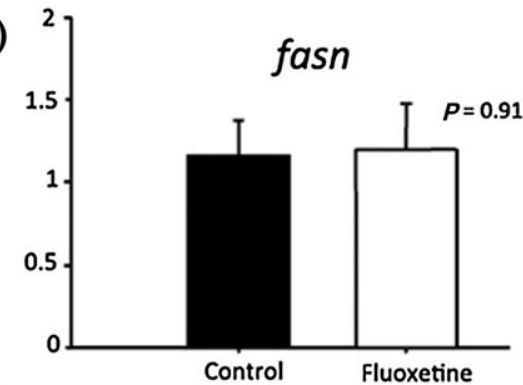

(D)

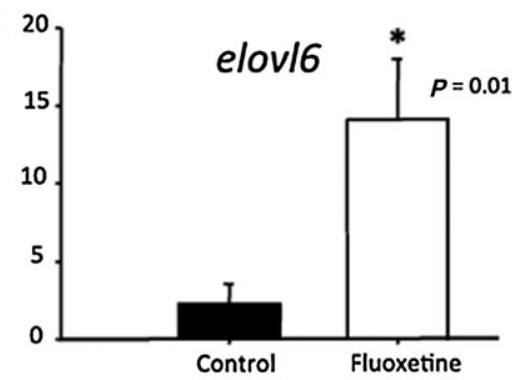

(F)

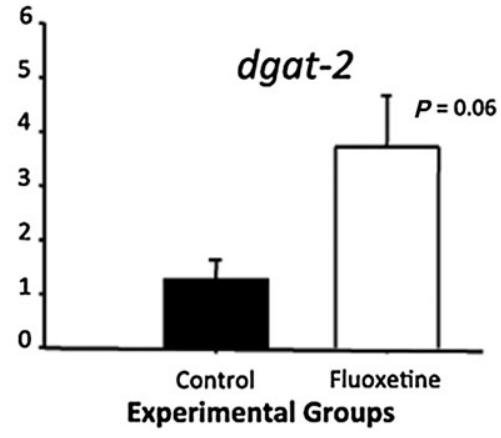

FIGURE 1 The effect of fetal and neonatal fluoxetine exposure on hepatic steady-state mRNA levels of Acc1, Fasn, Scd1, Elovl6, Dgat1 and Dgat2 in male rat offspring at 26 weeks of age. Data are presented as fold change from the controls and results are expressed as the mean \pm SEM. Values marked with an asterisk $\left({ }^{*}\right)$ are significantly $(P<0.05)$ different from controls ( $N=9-10$ per experimental group) 
Quantitative real-time PCR was employed using primers against the proximal rat promoters of Asc $(-155$ to +13$)$, Nlrp3 $(-180$ to -50), Casp1 (-155 to -62 ) and Elovl6 (-293 to -186) (Table 2; PE Applied Biosystems, Boston, Massachusetts). The transcriptional start sites (TSS) of each gene was found using the Ensembl Genome Browser (http://www.ensembl.org). The aforementioned constructed ChIP primers were then used in conjunction with Sso-Fast EvaGreen Supermix (Bio-Rad, Mississauga, Ontario) to perform qRT-PCR. Using serial dilutions of rat liver chromosomal DNA, the primers were demonstrated to have good linear correlation (slope $\sim 3.4$ ), suggesting equal priming efficiency in priming to their target sequence. Similar to the gene expression assays, the relative abundance of the immunoprecipitated chromatin compared to input chromatin was determined using the $2^{\Delta \Delta C t}$ method. The relative abundance of each primer set compared with calibrator was determined by the formula, $2 \Delta \Delta \mathrm{Ct}$, in which $\Delta \Delta \mathrm{Ct}$ is the calibrated $\mathrm{Ct}$ value.

\section{4 | Statistical analysis}

All statistical analyzes were performed using SigmaStat (v.3.1; SPSS, Chicago, Illinois). The results are expressed as the mean \pm SEM. The results from quantitative RT-PCR, immunoblot and ChIP analysis are expressed as the mean of arbitrary values \pm SEM. Outcome measures between male control and fluoxetine-treated offspring were analyzed using Student's t-test $(\alpha=0.05)$. Data were tested for normality as well as equal variance, and when normality or variance tests failed, data were analyzed using the Mann-Whitney rank sum test.

\section{3 | RESULTS}

\section{1 | De novo lipogenesis}

To elucidate the molecular mechanisms underlying the previously reported inflammation, elevated hepatic triglyceride levels, and NASH in fluoxetine-exposed male adult rat offspring, we examined the expression of key enzymes in de novo triglyceride production in the liver. In 26-week male fluoxetine-exposed offspring, quantitative real-time PCR revealed increases in the hepatic expression of Elovl6, Dgat1 and Dgat2, enzymes involved in the terminal steps of triglyceride synthesis, although the change in Dgat2 expression did not reach statistical significance $(P=0.06)$. In contrast, the expression of Acc1, Fasn, and Scd1, important enzymes for the initial stages of triglyceride synthesis were not significantly altered by early life exposure to fluoxetine (Figure 1).

(A)

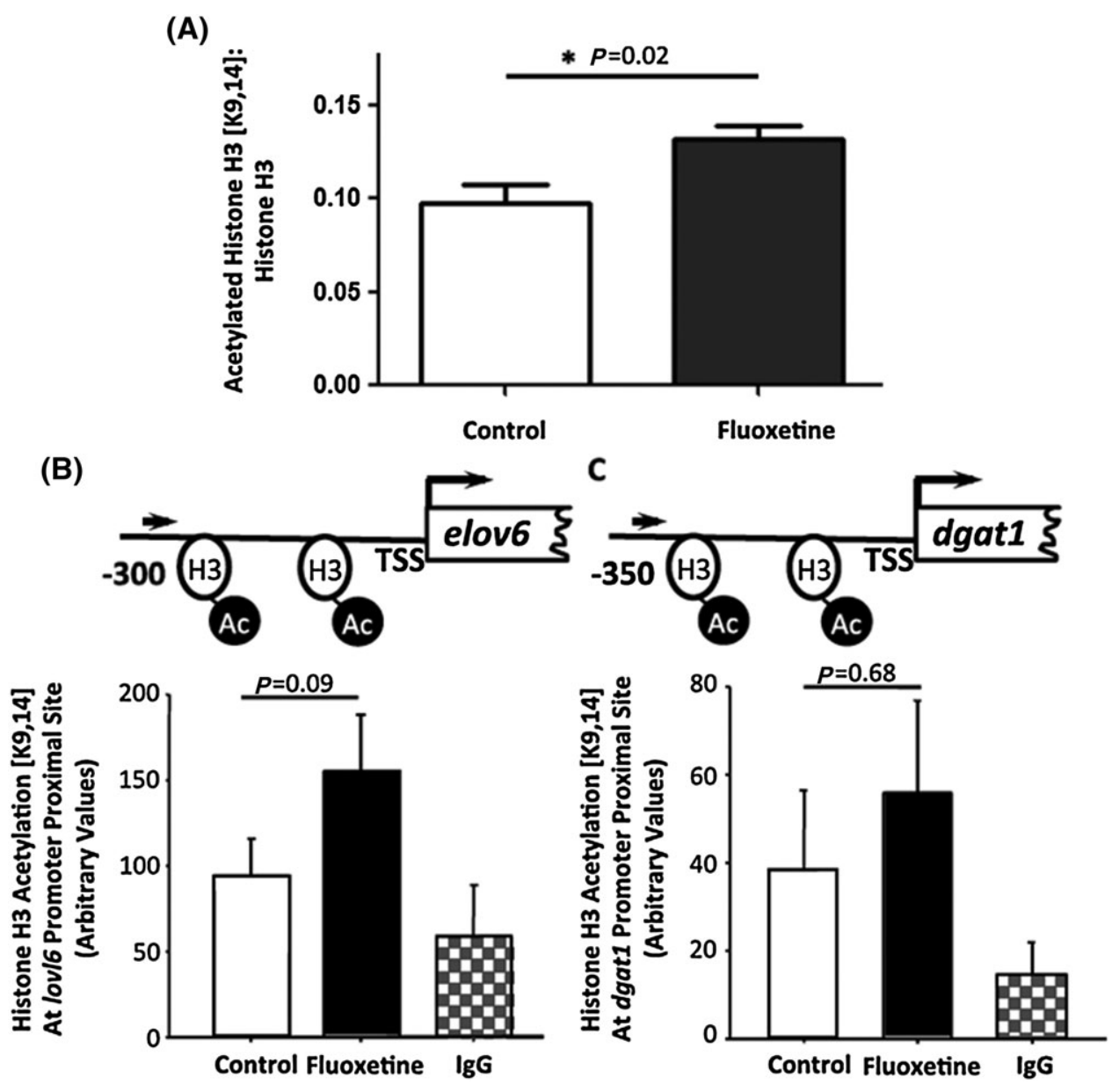

FIGURE 2 The effect of fetal and neonatal fluoxetine exposure on global and promoter-specific hepatic histone H3 [K9,14] acetylation in male rat offspring at 26 weeks of age. Data are presented as A, Densitometry of acetylated histone H3 [K9,14] to total histone H3, and B, ChIP analysis of relative histone $\mathrm{H} 3[\mathrm{~K} 9,14]$ immunoprecipitated genomic DNA levels normalized to total genomic DNA. Results are expressed at the mean \pm SEM. Values marked with an asterisk $\left({ }^{*}\right)$ are significantly $(P<0.05)$ different from controls $(N=6-8$ per experimental group) 


\subsection{Epigenetic regulation of de novo lipogenesis}

To explore whether fetal and neonatal fluoxetine exposure had any effect on post-translational histone modifications, we first investigated whether there were any global changes in total hepatic acetylated histone $\mathrm{H} 3$ lysine $9[\mathrm{~K} 9,14]$ in the offspring of fluoxetine-treated dams at 26 weeks of age. Immunoblotting revealed there was a significant increase in the global acetylation of $\mathrm{H} 3$ lysine $9[\mathrm{~K} 9,14]$ in the isolated histones from the livers of fluoxetine-exposed offspring at 26 weeks (Figure 2A).

Epigenetic posttranslational histone modifications such as increased histone $\mathrm{H} 3$ acetylation [K9,14], promoting chromatin opening, lead to transcriptional activation (Lee et al., 1993). Therefore, we next employed chromatin immunoprecipitation (ChIP) to determine whether this increased acetylation of histone $\mathrm{H} 3$ was associated, in a promoter-specific manner, with the observed increased transcription of Elovl6 and/or Dgat1. Although there was a trend $(P=0.09)$ for fluoxetine-exposed offspring to have higher $\mathrm{H} 3$ acetylation at the Elovl6 promoter, there was no significant difference in the histone $\mathrm{H} 3$ acetylation in the proximal promoter of either gene (Figure 2B and C).

\subsection{Increase in hepatic Elovl6 expression is} associated with a corresponding increase in the steady state mRNA levels of core components of the NLRP3 inflammasome in fluoxetine exposed offspring

In animal models, hepatic triglyceride accumulation has been associated with activation of the NLRP3 inflammasome (Yang, Lee, \& Lee, 2016). Interestingly, overexpression of Elovl6 in mouse hepatocytes has also been shown to upregulate the steady-state mRNA expression of NLRP3, ASC and caspase-1, core components of the NLRP3 inflammasome (Matsuzaka et al., 2012). Moreover, the product of Elovl6, stearate, has been demonstrated to activate the NLRP3 inflammasome in macrophages (L'homme et al., 2013). Therefore, we next employed real-time PCR to determine whether or not the increase in Elovl6 in fluoxetine-exposed offspring was associated with evidence of enhanced NLRP3 inflammasome expression. Despite the almost fivefold difference in NLRP3 mRNA expression between control and fluoxetine exposed offspring, this did not reach statistical significance $(P=0.10)$. However, fluoxetine exposure did result in a significant $(P \leq 0.05)$ upregulation of the steady state mRNA levels of the other core components of the NLRP3 inflammasome, ASC and caspase-1 (Figure 3).

\subsection{Augmented expression of ASC coincides with increased histone acetylation in the promoter region}

To investigate if chromatin remodeling could be a factor influencing the observed increases in NLRP3, ASC and caspase-1 steady-state mRNA levels after early life exposure to fluoxetine, we employed ChIP to assess histone $\mathrm{H} 3[\mathrm{~K} 9,14]$ acetylation in the promoters of these genes. ChIP revealed that in the 26-week livers of fluoxetine-exposed offspring there was significant enrichment in histone $\mathrm{H} 3[\mathrm{~K} 9,14]$ acetylation surrounding the proximal promoter of Asc but not Nlrp3 or Casp1 (Figure 4).
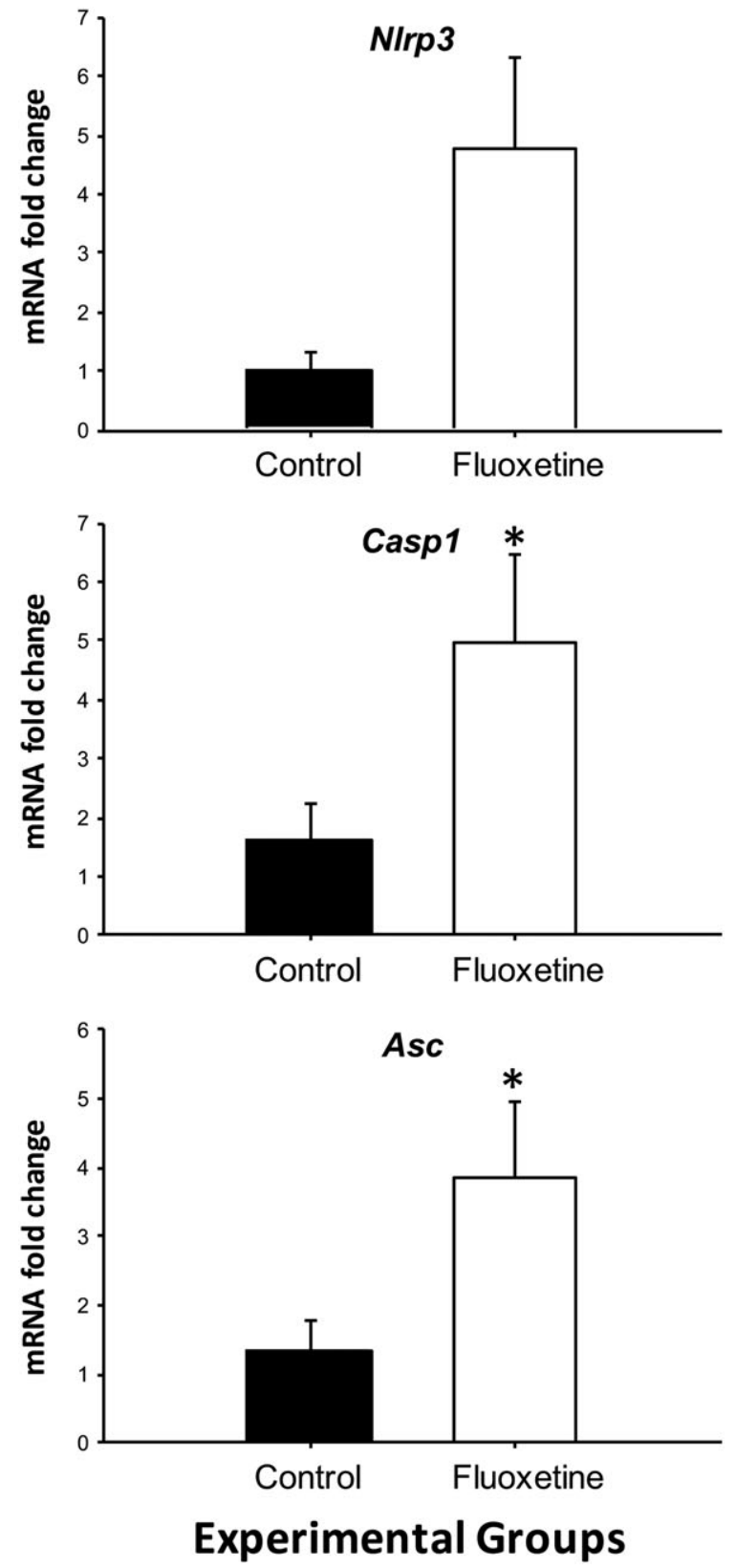

FIGURE 3 The effect of fetal and neonatal fluoxetine exposure on hepatic steady-state mRNA levels of NIrp3, Casp1 and Asc in male rat offspring at 26 weeks of age. Data are presented as fold change from the controls and results are expressed as the mean \pm SEM. Values marked with an asterisk $\left(^{*}\right)$ are significantly $(P<0.05)$ different from controls ( $N=9-10$ per experimental group)

\section{4 | DISCUSSION}

NAFLD is a major cause of liver disease worldwide, with an estimated global prevalence of approximately $25 \%$ (Younossi et al., 2016). It is a non-reversible condition which is characterized by hepatic steatosis, in particular, an accumulation of intrahepatic triglycerides (Tiniakos et al., 2010). Approximately $10-20 \%$ of patients with NAFLD go on to develop NASH which is characterized by steatosis plus hepatic inflammation and/or fibrosis (Tilg \& Moschen, 2010). Although the increasing global burden of NAFLD has significant implications for public health and health care expenditures, the pathophysiology of 


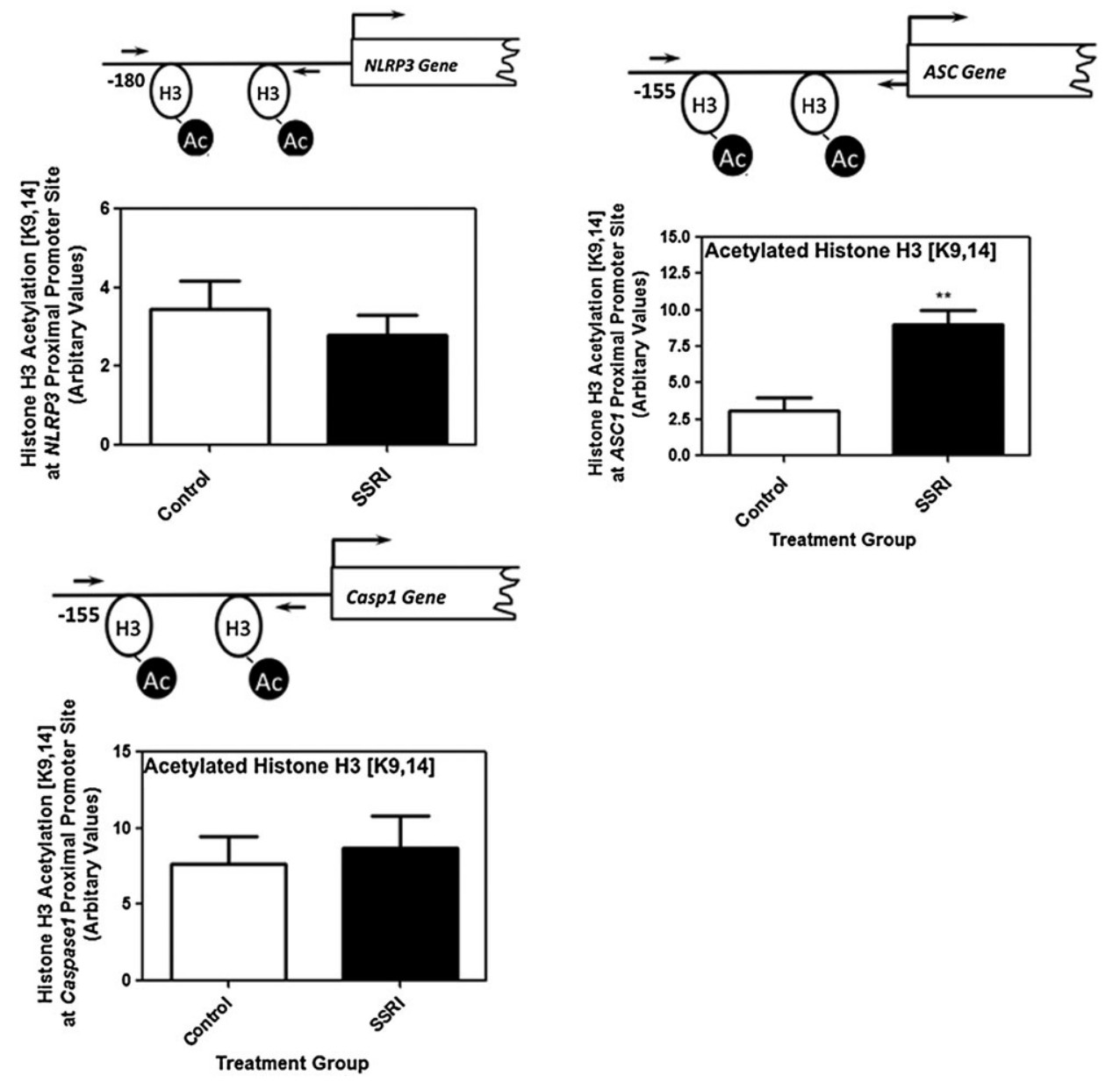

FIGURE 4 The effect of fetal and neonatal fluoxetine exposure on inflammasome promoter-specific hepatic histone H3 [K9,14] acetylation in male rat offspring at 26 weeks of age. Data are presented as ChIP analysis of relative histone H3 [K9,14] immunoprecipitated genomic DNA levels normalized to total genomic DNA. Results are expressed at the mean \pm SEM. Values marked with an asterisk $\left(^{*}\right)$ are significantly $(P<0.05)$ different from controls ( $N=6-8$ per experimental group)

NAFLD is not completely understood. However, increasing evidence from animal models suggest that chemical insults during fetal and neonatal development, including maternal smoking and ethanol consumption, lead to an increased risk of NAFLD in postnatal life (Lynch, Chan, $\&$ Drake, 2017). Similarly, work from our group has reported that fetal and neonatal exposure to fluoxetine leads to NAFLD in the offspring (De Long et al., 2015). Given that approximately $6 \%$ of pregnant women in the United States are prescribed SSRIs during pregnancy (Andrade, Reichman, Mott, \& Pitts, 2016), this highlights the importance of understanding the effects of these medications on the risk of NAFLD in the offspring.

Accumulation of hepatic intracytoplasmic triglycerides is a key component of NAFLD (Tiniakos et al., 2010) and results from an imbalance between lipid uptake, synthesis, utilization and export (Tiniakos et al., 2010). However, data from humans have shown that increased de novo lipogenesis may be the critical factor in the development of NAFLD (Diraison et al., 2003; Donnelly et al., 2005; Lambert, RamosRoman, Browning, \& Parks, 2014; Softic et al., 2016). In our animal model, fetal and neonatal exposure to fluoxetine increased hepatic triglyceride content without a concomitant increase in circulating triglyceride levels (De Long et al., 2015). Moreover, cell culture studies have revealed that fluoxetine treatment increases genes critical for de novo lipogenesis (Feng et al., 2012; Xiong et al., 2014). Taken together, these data suggested that the observed increase in hepatic triglycerides in this perinatal exposure model might be mediated by upregulation of de novo lipogenesis. Indeed, results from the current study supported this hypothesis; steady-state mRNA levels of Elovl6 and Dgat1 were elevated in the liver of male fluoxetine-exposed offspring at 26 weeks of age.

Elovl6 is an enzyme responsible for catalyzing the elongation of unsaturated and monounsaturated fatty acid chains C:12-C:18 (Jakobsson, Westerberg, \& Jacobsson, 2006) whereas Dgat1 catalyzes the terminal step in triglyceride synthesis (Liu, Siloto, Lehner, Stone, \& Weselake, 2012). In mouse models, over-expression of both Dgat1 and Elovl6 has been reported to result in increased hepatic triglyceride content (Matsuzaka et al., 2012; Monetti et al., 2007). Similarly, in our model the increased expression of Elovl6 and Dgat1 in fluoxetineexposed offspring occurred in concert with increased liver triglyceride content (De Long et al., 2015). Moreover, in mice, overexpression of Elovl6 but not Dgat1 was associated with hepatic inflammation (Monetti et al., 2007; Matsuzaka et al., 2012;). Collectively, these data suggest that increased expression of hepatic Elovl6 may play a key role in etiology of NASH in fluoxetine-exposed offspring. Further support for this hypothesis comes from a human study where hepatic Elovl6 mRNA expression was increased in patients with NASH compared to control subjects (Matsuzaka et al., 2012). The mechanisms linking 
increased hepatic Elovl6 expression to NASH have not been fully elucidated, but evidence from animal and cell culture models suggests that it may involve the NLRP3 inflammasome.

Expression of NLRP3 and its key components have been shown to be increased in rodents and humans with NASH (Wan et al., 2016). Moreover, pharmacological inhibition of the NLRP3 inflammasome suppressed high-fat diet-induced hepatic steatosis (Yang et al., 2016), implicating that this is a causal relationship. In contrast, overexpression of Elovl6 in mice activated the NLRP3 inflammasome (Matsuzaka et al., 2012). Similarly, in our study increased Elovl6 mRNA expression in fluoxetine-exposed animals was associated with increased expression of NLRP3, ASC and caspase-1, although the increase in NLRP3 did not reach statistical significance $(P=0.10)$. Taken together these data are consistent with a link between dysregulation of Elovl6, activation of the NLRP3 inflammasome and NASH in fluoxetine-exposed offspring. However, it is noteworthy that the increase in core components of the hepatic NLRP3 inflammasome after early life exposure to fluoxetine animals is at odds with studies in adult animals where fluoxetine has been shown to directly inhibit NLRP3 inflammasome activity in peripheral macrophages, microglia and the prefrontal cortex (Du et al., 2016; Pan, Chen, Zhang, \& Kong, 2014). Whether this is related to the timing of exposure or represents a tissue-specific effect remains to be determined. To further explore the underlying molecular mechanisms by which early life exposure to fluoxetine could affect de novo lipogenesis and/or the NLRP3 inflammasome, we decided to investigate the role of epigenetic modifications in our model.

There is now considerable evidence to suggest that chemical insults during perinatal development can increase the risk of metabolic syndrome in the postnatal life via alterations in the epigenome (Desai, Jellyman, \& Ross, 2015). These epigenetic modifications can include altered DNA methylation, histone modifications, chromatin remodeling and/or changes in microRNAs. Maternal fluoxetine exposure has been shown to result in posttranslational histone modifications and altered DNA methylation in the offspring (Boulle et al., 2016; Toffoli et al., 2014). There is also some evidence in the literature that demonstrates epigenetic regulation of Elovl6 and core components of NLRP3 inflammasome (Corominas et al., 2015; Meier, Drexler, Eberle, Lefort, \& Yazdi, 2016; Tang et al., 2016). Moreover, knockdown of sirtuin 1 (SIRT1), a NAD ${ }^{+}$-dependent deacetylase has been demonstrated to enhance activation of the NLRP3 inflammasome in HUVEC cells (Li et al., 2017) and inhibition of SIRT1 in hepatocytes results in an upregulation of de novo lipogenesis related genes (Nassir \& Ibdah, 2016). Taken together these data suggest that post-translational histone modifications may also represent an important pathway by which early life exposure to fluoxetine can increase the risk of NASH. Remarkably, we demonstrated that fetal and neonatal exposure to fluoxetine results in increased global acetylated histone $\mathrm{H} 3$ in the liver of male offspring at 26 weeks of age. Despite no changes in histone H3 acetylation [K9,14] in the promoters of Elovl6, Dgat, Nlrp3 or Casp1, the increase in Asc expression was concomitant with enhanced histone $\mathrm{H} 3$ acetylation $[K 9,14]$ at surrounding its proximal promoter region which would promote transcriptional activation. This is of great interest considering epigenetic regulation of Asc appears to be key factor for the activation of the inflammasome complex. For example, a study in human cutaneous squamous cell carcinoma reported that promoter specific methylation of Asc led to a decrease in its expression concomitant with impaired activation of the NLRP3 inflammasome (Meier et al., 2016). Therefore it is tempting to speculate that increased expression of Asc also plays a key role in the development of hepatic inflammation in our model, although evidence for a causal relationship remains to be further explored.

In summary, we have shown for the first time that fetal and neonatal exposure to fluoxetine increases hepatic expression of Elovl6 and core components of the NLRP3 inflammasome which may explain, in part, the increased prevalence of NASH in these animals. However, it is impossible to determine from the present study whether this phenotype was present from the time of exposure or whether there was latency in its onset. Regardless, given the widespread usage of these drugs during pregnancy, these results suggest that evaluation of metabolic outcomes in children exposed to SSRI antidepressants during fetal and neonatal life warrants further study.

\section{CONFLICT OF INTEREST}

The authors did not report any conflict of interest.

\section{FUNDING}

This work was supported by the Canadian Institutes of Health Research (MOP119323 to ACH and MOP111001 to DBH). Salary support for NED was provided by the CIHR Training Program in Reproduction, Early Development and the Impact on Health (REDIH).

\section{REFERENCES}

Alboni, S., Poggini, S., Garofalo, S., Milior, G., Hajj, El, H., Lecours, C., ... Branchi, I. (2016). Fluoxetine treatment affects the inflammatory response and microglial function according to the quality of the living environment. Brain, Behavior, and Immunity, 58, 261-271. https://doi. org/10.1016/j.bbi.2016.07.155

Andrade, S. E., Raebel, M. A., Brown, J., Lane, K., Livingston, J., Boudreau, D., ... Platt, R. (2008). Use of antidepressant medications during pregnancy: A multisite study. American Journal of Obstetrics and Gynecology, 198, 194.e1-194.e5. https://doi.org/10.1016/j. ajog.2007.07.036

Andrade, S. E., Reichman, M. E., Mott, K., \& Pitts, M. (2016). Use of selective serotonin reuptake inhibitors (SSRIs) in women delivering liveborn infants and other women of child-bearing age within the U.S. Food and Drug Administration's mini-sentinel program. Archives of Women's Mental Health, 19, 969-977.

Bellentani, S. (2017). The epidemiology of non-alcoholic fatty liver disease. Liver International, 37, 81-84. https://doi.org/10.1111/liv.13299

Boulle, F., Pawluski, J. L., Homberg, J. R., Machiels, B., Kroeze, Y., Kumar, N., ... Van den Hove, D. L. A. (2016). Developmental fluoxetine exposure increases behavioral despair and alters epigenetic regulation of the hippocampal BDNF gene in adult female offspring. Hormones and Behavior, 80, 47-57. https://doi.org/10.1016/j.yhbeh.2016.01.017

Burt, V. K., \& Stein, K. (2002). Epidemiology of depression throughout the female life cycle. The Journal of Clinical Psychiatry, 63, 9-15.

Capello, C. F., Bourke, C. H., Ritchie, J. C., Stowe, Z. N., Newport, D. J., Nemeroff, A., \& Owens, M. J. (2011). Serotonin transporter occupancy in rats exposed to serotonin reuptake inhibitors in utero or via breast milk. The Journal of Pharmacology and Experimental Therapeutics, 339, 275-285. https://doi.org/10.1124/jpet.111.183855

Cooper, W. O., Willy, M. E., Pont, S. J., \& Ray, W. A. (2007). Increasing use of antidepressants in pregnancy. American Journal of Obstetrics and Gynecology, 196, 544. e1-544. e5. 
Corominas, J., Marchesi, J. A., Puig-Oliveras, A., Revilla, M., Estellé, J., Alves, E., ... Ballester, M. (2015). Epigenetic regulation of the ELOVL6 gene is associated with a major QTL effect on fatty acid composition in pigs. Genetics, Selection, Evolution, 47, 45358. https://doi.org/10.1186/ s12711-015-0111-y

De Long, N. E., Barry, E. J., Pinelli, C., Wood, G. A., Hardy, D. B., Morrison, K. M., ... Holloway, A. C. (2015). Antenatal exposure to the selective serotonin reuptake inhibitor fluoxetine leads to postnatal metabolic and endocrine changes associated with type 2 diabetes in Wistar rats. Toxicology and Applied Pharmacology, 285, 32-40. https://doi.org/ 10.1016/j.taap.2015.03.006

De Nardo, D., \& Latz, E. (2011). NLRP3 inflammasomes link inflammation and metabolic disease. Trends in Immunology, 32, 373-379. https:// doi.org/10.1016/j.it.2011.05.004

Desai, M., Jellyman, J. K., \& Ross, M. G. (2015). Epigenomics, gestational programming and risk of metabolic syndrome. International Journal of Obesity, 39, 633-641. https://doi.org/10.1038/ijo.2015.13

Diraison, F., Moulin, P., \& Beylot, M. (2003). Contribution of hepatic de novo lipogenesis and reesterification of plasma non esterified fatty acids to plasma triglyceride synthesis during non-alcoholic fatty liver disease. Diabetes \& Metabolism, 29, 478-485.

Donnelly, K. L., Smith, C. I., Schwarzenberg, S. J., Jessurun, J., Boldt, M. D., \& Parks, E. J. (2005). Sources of fatty acids stored in liver and secreted via lipoproteins in patients with nonalcoholic fatty liver disease. The Journal of Clinical Investigation, 115, 1343-1351. https://doi.org/ $10.1172 / \mathrm{JCl} 23621$

Du, R. H., Tan, J., Sun, X. Y., Lu, M., Ding, J. H., \& Hu, G. (2016). Fluoxetine inhibits NLRP3 Inflammasome activation: Implication in depression. The International Journal of Neuropsychopharmacology, 19. https://doi.org/ 10.1093/ijnp/pyw037

Duwaerts, C. C., \& Maher, J. J. (2014). Mechanisms of liver injury in nonalcoholic steatohepatitis. Current Hepatology Reports, 13, 119-129. https://doi.org/10.1007/s11901-014-0224-8

Feng, X. M., Xiong, J., Qin, H., Liu, W., Chen, R. N., Shang, W., ... Yang, J. (2012). Fluoxetine induces hepatic lipid accumulation via both promotion of the SREBP1c-related lipogenesis and reduction of lipolysis in primary mouse hepatocytes. CNS Neuroscience \& Therapeutics, 18, 974-980. https://doi.org/10.1111/cns.12014

Gavin, N. I., Gaynes, B. N., Lohr, K. N., Meltzer-Brody, S., Gartlehner, G., \& Swinson, T. (2005). Perinatal depression: A systematic review of prevalence and incidence. Obstetrics and Gynecology, 106, 1071-1083. https://doi.org/10.1097/01.AOG.0000183597.31630.db

Gelaye, B., Rondon, M. B., Araya, R., \& Williams, M. A. (2016). Epidemiology of maternal depression, risk factors, and child outcomes in low-income and middle-income countries. Lancet Psychiatry, 3, 973-982. https:// doi.org/10.1016/S2215-0366(16)30284-X

Grote, N. K., Bridge, J. A., Gavin, A. R., Melville, J. L., lyengar, S., \& Katon, W. J. (2010). A meta-analysis of depression during pregnancy and the risk of preterm birth, low birth weight, and intrauterine growth Restriction. Archives of General Psychiatry, 67, 1012-1024. https://doi.org/ 10.1001/archgenpsychiatry.2010.111

Grzeskowiak, L. E., Gilbert, A. L., Sørensen, T. I. A., Olsen, J., Sørensen, H. T., Pedersen, L. H., \& Morrison, J. L. (2013). Prenatal exposure to selective serotonin reuptake inhibitors and childhood overweight at 7 years of age. Annals of Epidemiology, 23, 681-687. https://doi.org/10.1016/j. annepidem.2013.08.005

Haneklaus, M., \& O'Neill, L. A. J. (2015). NLRP3 at the interface of metabolism and inflammation. Immunological Reviews, 265, 53-62. https:// doi.org/10.1111/imr.12285

Hui, Y., Huang, N., Ebbert, L., \& Bina, H. (2007). Pharmacokinetic comparisons of tail-bleeding with cannula-or retro-orbital bleeding techniques in rats using six marketed drugs. Journal of Pharmacological and Toxicological Methods, 56, 256-264.

Huybrechts, K. F., Palmsten, K., Mogun, H., Kowal, M., Avorn, J., SetoguchiIwata, S., \& Hernández-Díaz, S. (2013). National trends in antidepressant medication treatment among publicly insured pregnant women.
General Hospital Psychiatry, 35, 265-271. https://doi.org/10.1016/j. genhosppsych.2012.12.010

Jakobsson, A., Westerberg, R., \& Jacobsson, A. (2006). Fatty acid elongases in mammals: Their regulation and roles in metabolism. Progress in Lipid Research, 45, 237-249. https://doi.org/10.1016/j.plipres.2006.01.004

Jerrell, J. M. (2010). Neuroendocrine-related adverse events associated with antidepressant treatment in children and adolescents. CNS Neuroscience \& Therapeutics, 16, 83-90. https://doi.org/10.1111/j.17555949.2009.00106.x

Jo, E. K., Kim, J. K., Shin, D. M., \& Sasakawa, C. (2016). Molecular mechanisms regulating NLRP3 inflammasome activation. Cellular \& Molecular Immunology, 13, 148-159. https://doi.org/10.1038/cmi.2015.95

Lambert, J. E., Ramos-Roman, M. A., Browning, J. D., \& Parks, E. J. (2014). Increased de novo lipogenesis is a distinct characteristic of individuals with nonalcoholic fatty liver disease. Gastroenterology, 146, 726-735. https://doi.org/10.1053/j.gastro.2013.11.049

L'homme, L., Esser, N., Riva, L., Scheen, A., Paquot, N., Piette, J., \& LegrandPoels, S. (2013). Unsaturated fatty acids prevent activation of NLRP3 inflammasome in human monocytes/macrophages. Journal of Lipid Research, 54, 2998-3008. https://doi.org/10.1194/jlr.M037861

Li, Y., Yang, X., He, Y., Wang, W., Zhang, J., Zhang, W., ... Lin, R. (2017) Negative regulation of NLRP3 inflammasome by SIRT1 in vascular endothelial cells. Immunobiology, 222, 552-561. https://doi.org/ 10.1016/j.imbio.2016.11.002

Liu, Q., Siloto, R. M. P., Lehner, R., Stone, S. J., \& Weselake, R. J. (2012) Acyl-CoA:Diacylglycerol acyltransferase: Molecular biology, biochemistry and biotechnology. Progress in Lipid Research, 51, 350-377. https:// doi.org/10.1016/j.plipres.2012.06.001

Livak, K. J., \& Schmittgen, T. D. (2001). Analysis of relative Gene expression data using real-time quantitative PCR and the 2- $\triangle \mathrm{CT}$ method Methods, 25, 402-408. https://doi.org/10.1006/meth.2001.1262

Lynch, C., Chan, C. S., \& Drake, A. J. (2017). Early life programming and the risk of non-alcoholic fatty liver disease. Journal of Developmental Origins of Health and Disease, 23, 1-10. https://doi.org/10.1017/ S2040174416000805

Marcus, S. M. (2009). Depression during pregnancy: Rates, risks and consequences--Motherisk update 2008. The Canadian Journal of Clinical Pharmacology, 16, e15-e22.

Martinon, F., Burns, K., \& Tschopp, J. (2002). The inflammasome: A molecular platform triggering activation of inflammatory caspases and processing of prolL-beta. Molecular Cell, 10, 417-426.

Matsuzaka, T., Atsumi, A., Matsumori, R., Nie, T., Shinozaki, H., SuzukiKemuriyama, N., ... Shimano, H. (2012). Elovl6 promotes nonalcoholic steatohepatitis. Hepatology, 56, 2199-2208. https://doi.org/10.1002/ hep. 25932

Meier, K., Drexler, S. K., Eberle, F. C., Lefort, K., \& Yazdi, A. S. (2016). Silencing of ASC in cutaneous squamous cell carcinoma. PloS One, 11. e0164742. https://doi.org/10.1371/journal.pone.0164742

Monetti, M., Levin, M. C., Watt, M. J., Sajan, M. P., Marmor, S., Hubbard, B. K., ... Farese, R. V. (2007). Dissociation of hepatic steatosis and insulin resistance in mice overexpressing DGAT in the liver. Cell Metabolism, 6, 69-78. https://doi.org/10.1016/j.cmet.2007.05.005

Muzik, M., \& Hamilton, S. E. (2016). Use of antidepressants during pregnancy?: What to consider when weighing treatment with antidepressants against untreated depression. Maternal and Child Health Journal, 20, 2268-2279. https://doi.org/10.1007/s10995-0162038-5

Nassir, F., \& Ibdah, J. A. (2016). Sirtuins and nonalcoholic fatty liver disease. World Journal of Gastroenterology, 22, 10084-10092. https://doi.org/ 10.3748/wjg.v22.i46.10084

Noble, R. E. (2005). Depression in women. Metabolism, 54, 49-52. https:// doi.org/10.1016/j.metabol.2005.01.014

Olivier, J. D. A., Vallès, A., van Heesch, F., Afrasiab-Middelman, A., Roelofs, J. J. P. M., Jonkers, M., ... Homberg, J. R. (2011). Fluoxetine administration to pregnant rats increases anxiety-related behavior in the offspring. 
Psychopharmacology, 217, 419-432. https://doi.org/10.1007/s00213011-2299-z

Pan, Y., Chen, X. Y., Zhang, Q. Y., \& Kong, L. D. (2014). Microglial NLRP3 inflammasome activation mediates IL-1 $\beta$-related inflammation in prefrontal cortex of depressive rats. Brain, Behavior, and Immunity, 41, 90-100. https://doi.org/10.1016/j.bbi.2014.04.007

Rampono, J., Simmer, K., Ilett, K. F., Hackett, L. P., Doherty, D. A., Elliot, R., ... Forman, T. (2009). Placental transfer of SSRI and SNRI antidepressants and effects on the neonate. Pharmacopsychiatry, 42, 95-100. https://doi.org/10.1055/s-0028-1103296

Schroder, K., \& Tschopp, J. (2010). The inflammasomes. Cell, 140, 821-832. https://doi.org/10.1016/j.cell.2010.01.040

Schwartz, B. S., Glass, T. A., Pollak, J., Hirsch, A. G., Bailey-Davis, L., Moran, T. H., \& Bandeen-Roche, K. (2016). Depression, its comorbidities and treatment, and childhood body mass index trajectories. Obesity, 24, 2585-2592. https://doi.org/10.1002/oby.21627

Sit, D. K., Perel, J. M., Helsel, J. C., \& Wisner, K. L. (2008). Changes in antidepressant metabolism and dosing across pregnancy and early postpartum. The Journal of Clinical Psychiatry, 69, 652-658.

Smith, A. J., Sketris, I., Cooke, C., Gardner, D., Kisely, S., \& Tett, S. E. (2008). A comparison of antidepressant use in Nova Scotia, Canada and Australia. Pharmacoepidemiology and Drug Safety, 17, 697-706. https://doi.org/10.1002/pds.1541

Softic, S., Cohen, D. E., \& Kahn, C. R. (2016). Role of dietary fructose and hepatic de novo lipogenesis in fatty liver disease. Digestive Diseases and Sciences, 61, 1282-1293. https://doi.org/10.1007/s10620-0164054-0

Sohi, G., Marchand, K., Revesz, A., Arany, E., \& Hardy, D. B. (2011). Maternal protein Restriction elevates Cholesterol in adult rat offspring due to repressive changes in histone modifications at the Cholesterol 7aHydroxylasePromoter. Molecular Endocrinology, 25, 785-798. https:// doi.org/10.1210/me.2010-0395

Stein, A., Pearson, R. M., Goodman, S. H., Rapa, E., Rahman, A., McCallum, M., \& ...Pariante CM. (2014). Effects of perinatal mental disorders on the fetus and child. Lancet, 384, 1800-1819. https://doi.org/ 10.1016/S0140-6736(14)61277-0

Tang, S. C., Yeh, J. I., Hung, S. J., Hsiao, Y. P., Liu, F. T., \& Yang, J. H. (2016). Glycolic acid silences Inflammasome complex genes, NLRC4 and ASC, by inducing DNA methylation in HaCaT cells. DNA and Cell Biology, 35, 124-134. https://doi.org/10.1089/dna.2015.2993

Tilg, H., \& Moschen, A. R. (2010). Evolution of inflammation in nonalcoholic fatty liver disease: The multiple parallel hits hypothesis. Hepatology, 52 1836-1846. https://doi.org/10.1002/hep.24001

Tiniakos, D. G., Vos, M. B., \& Brunt, E. M. (2010). Nonalcoholic fatty liver disease: Pathology and pathogenesis. Annual Review of Pathology, 5, 145-171. https://doi.org/10.1146/annurev-pathol-121808-102132
Toffoli, L. V., Rodrigues, G. M., Oliveira, J. F., Silva, A. S., Moreira, E. G. Pelosi, G. G., \& Gomes, M. V. (2014). Maternal exposure to fluoxetine during gestation and lactation affects the DNA methylation programming of rat's offspring: Modulation by folic acid supplementation. Behavioural Brain Research, 265, 142-147. https://doi.org/10.1016/j. bbr.2014.02.031

Trifirò, G., Tillati, S., Spina, E., Ferrajolo, C., Alacqua, M., Aguglia, E., ... Samani, F. (2013). A nationwide prospective study on prescribing pattern of antidepressant drugs in Italian primary care. European Journal of Clinical Pharmacology, 69, 227-236. https://doi.org/10.1007/ s00228-012-1319-1

Wan, X., Xu, C., Yu, C., \& Li, Y. (2016). Role of NLRP3 Inflammasome in the progression of NAFLD to NASH. Canadian Journal of Gastroenterology and Hepatology, 2016. 6489012. https://doi.org/10.1155/2016/ 6489012

Wemakor, A., Casson, K., \& Dolk, H. (2014). Prevalence and sociodemographic patterns of antidepressant use among women of reproductive age: A prescription database study. Journal of Affective Disorders, 167, 299-305. https://doi.org/10.1016/j.jad.2014.06.015

World Health Organization (2008). Mental Health Gap Action Programme: scaling up care for mental, neurological and substance use disorders. Geneva: World Health Organization.

Xiong, J., Yang, H., Wu, L., Shang, W., Shan, E., Liu, W., ... Yang, J. (2014). Fluoxetine suppresses AMP-activated protein kinase signaling pathway to promote hepatic lipid accumulation in primary mouse hepatocytes. The International Journal of Biochemistry \& Cell Biology, 54, 236-244. https://doi.org/10.1016/j.biocel.2014.07.019

Yang, G., Lee, H. E., \& Lee, J. Y. (2016). A pharmacological inhibitor of NLRP3 inflammasome prevents non-alcoholic fatty liver disease in a mouse model induced by high fat diet. Scientific Reports, 6, 26218. https://doi.org/10.1038/srep26218

Yoon, J. M., Cho, E. G., Lee, H. K., \& Park, S. M. (2013). Antidepressant use and diabetes mellitus risk: A meta-analysis. Korean Journal of Family Medicine, 34, 228-240. https://doi.org/10.4082/kjfm.2013.34.4.228

Younossi, Z. M., Koenig, A. B., Abdelatif, D., Fazel, Y., Henry, L., \& Wymer, M. (2016). Global epidemiology of nonalcoholic fatty liver diseasemeta-analytic assessment of prevalence, incidence, and outcomes. Hepatology, 64, 73-84. https://doi.org/10.1002/hep.28431

How to cite this article: De Long NE, Hardy $\mathrm{DB}, \mathrm{Ma} N$, Holloway AC. Increased incidence of non-alcoholic fatty liver disease in male rat offspring exposed to fluoxetine during fetal and neonatal life involves the NLRP3 inflammasome and augmented de novo hepatic lipogenesis. J Appl Toxicol. 2017;1-10. https://doi.org/10.1002/jat.3502 